\title{
VARIAÇÃO ESPACIAL DA PRECIPITAÇÃO DAS CIDADES DO CENTRO DO SUL DO ESTADO DO CEARÁ, BRASIL
}

\author{
Eldir Bandeira da Silva \\ Graduando em Tecnologia em Irrigação e Drenagem \\ eldir_2005@hotmail.com \\ Kleber Gomes de Macêdo \\ Graduando em Tecnologia em Irrigação e Drenagem \\ kleber117@hotmail.com
}

Anny Kariny Feitosa

Doutoranda em Ambiente e Desenvolvimento

akfeitosa@hotmail.com

José Ribeiro de Araújo Neto

Doutorando em Engenharia Agrícola

juniorifcelabas@gmail.com

\section{RESUMO}

A quantidade de chuva representada numa região é um dos fatores mais importantes para a caracterização do clima e, por conseguinte, nas relações de consumo da mesma. É importante também para o desenvolvimento socioeconômico de uma região, tendo em vista o seu diverso uso em todos os setores. Analisaram-se, neste trabalho, a espacialização da variabilidade da precipitação média num período de 10 anos das cidades do Centro Sul do Ceará com o objetivo de caracterizar quais cidades estão sofrendo com a estiagem/seca e o que deve ser feito para que este cenário mude. Os resultados obtidos foram de que numa pequena região há bastante oscilação quanto à precipitação, que grande parte dos municípios estudados estão abaixo da média gerada e que eventos como El Niño, La Niña e Zona de Convergência Intertropical (ZCIT) podem influenciar no regime pluviométrico quando sendo analisadas em conjunto, não separadamente. Também se concluiu que a espacialização dos dados, serve de maneira geral, para fins de dimensionamento agronômico e potencialização de pontos para armazenamento de água em lugares estratégicos.

Palavras-chaves: crise hídrica, variabilidade da precipitação, semiárido

\begin{abstract}
The amount of rainfall in a region represented one of the most important factors to characterize the climate and therefore in consumer relations thereof. It is also important for socio-economic development of a region, given its diverse use in all sectors. Were analyzed in this work, the spatial variability of the average rainfall in a 10-year period from cities in the south of Ceará Center in order to characterize which cities are suffering from the drought / dry and what should be done so that this scenario change. The results were that a small region there are plenty of variations on the rainfall, large parts of the cities studied are below average generated and that events such as El Niño, La Niña and Intertropical Convergence Zone (ITCZ) may influence the rainfall when being taken together, not separately. It was also found that the spatial distribution is in general use for agronomic purposes sizing and water storage in strategic places.
\end{abstract}

Keywords: water crisis, variability of precipitation, semiarid region. 


\section{INTRODUÇÃO}

A sazonalidade das chuvas na região Nordeste do Brasil, em conjunto com a baixa incidência pluviométrica, é um dos principais fatores dos eventos de estiagem na região (HASTENRATH, 1984). Entretanto, as características como a variabilidade espacial e temporal das chuvas são os fatores que afetam a sociedade de forma mais aguda (MAGALHÃES E GLANTZ, 1992).

O índice pluviométrico é um dos fatores principais para a caracterização do clima de qualquer localidade, por seus efeitos essenciais no desenvolvimento das culturas. O estudo torna-se imprescindível na gestão e no planejamento de atividades agrícolas permitindo previsões com melhores aproximações e decisões mais confiáveis (ARAI, 2010). Grande parte dos projetos de irrigação usam valores médios de precipitações para fins de dimensionamento. Porém, deve-se levar em consideração a distribuição como um todo, pois sabe-se que as precipitações são irregulares e ainda se pode analisar os tempos de retorno (FIETZ et al., 2002).

Para Somlyody \& Varis (2006), a grande problemática da crise de água é decorrente da falta de planejamento em estratégias de gerenciamento e armazenamento de água, com uma temática mais preventiva do que corretiva. Rogers et al. (2006) também discorre em seus trabalhos que o grande ponto de interrogação está no fato de que a água que é precipitada não é armazenada, sendo perdida por diversos fatores.

$\mathrm{Na}$ busca por espacialização dos dados das precipitações, é empregada através dos Sistemas de Informações Geográficas (SIGs), a distribuição das chuvas na região associada a diversos fatores climáticos, objetivando uma visão mais abrangente e detalhada da causa (FREITAS et al., 2001). Com isso, vários autores têm usado a interpolação para estimativa de sua espacialização climática. Contudo é necessário atenção para que tipo de interpolação se esteja usando, pois cada tipo se adéqua de maneira diferente (RIBEIRO DA SILVA et al., 2007).

O Ceará, pelo fato de estar inserido no semiárido nordestino, possui características próprias quanto aos fatores hidroclimáticos, sobretudo à distribuição das precipitações, elevadas taxas evapotranspirativas e rios intermitentes. A água ofertada para abastecimento na região é advinda dos reservatórios, sendo $93 \%$ da água consumida oriunda das barragens (MALVEIRA, 2009).

Diante do exposto, este trabalho tem como objetivo apresentar a espacialização dos índices pluviométricos das cidades do Centro Sul do Ceará para fins de dimensionamento e gestão hídrica, e para deteccão de cidades que estão sofrendo com a estiagem/seca.

\section{MATERIAL E MÉTODOS}

As cidades escolhidas para este estudo pertencerem a uma bacia com predominância de escassez hídrica e estão apresentadas na Figura 1. As mesmas estão localizadas na região Centro Sul do Ceará sobre as Bacias Hidrográficas do Alto Jaguaribe e Salgado, que juntas correspondem a 24,81\% do território cearense, e responsável por 31 açudes públicos monitorados pela COGERH. O clima em boa parte do território do Estado do Ceará é semiárido, com médias pluviométricas inferiores a $600 \mathrm{~mm}$ e irregularidade nas precipitações, o que ocasiona secas periódicas. Em consequência desse fenômeno, os cursos d'água são temporários, permanecendo secos ao longo de todo o verão, e a vegetação dominante é a da caatinga, com sua paisagem típica, de pequenas árvores retorcidas (SILVA et al., 2007). 


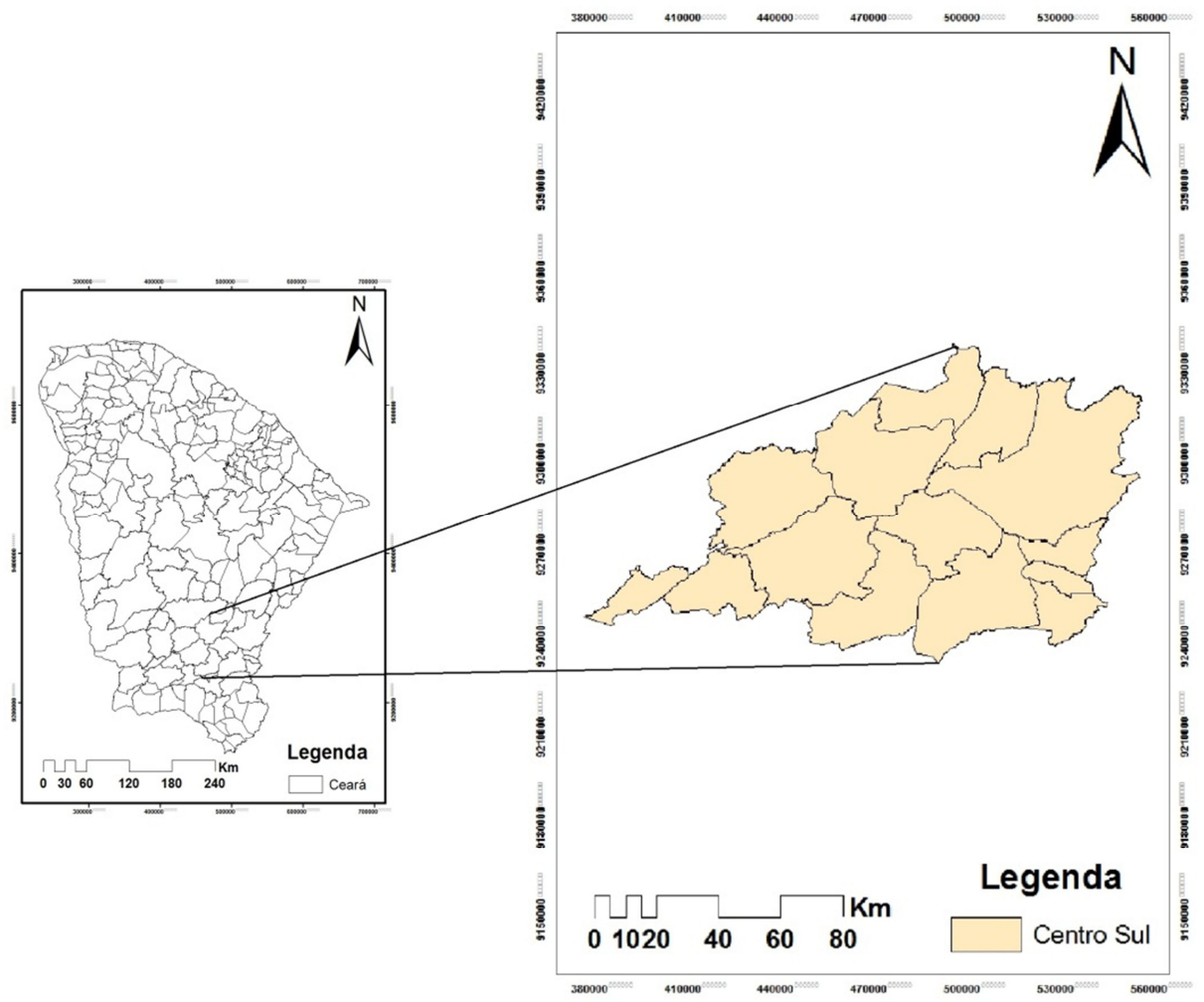

Figura 1. Representação a região Centro Sul do Estado do Ceará. Fonte: Elaboração própria.

Para a espacialização das precipitações pluviométricas da região em estudo foram utilizados dados históricos da Fundação Cearense de Meteorologia - FUNCEME. A Tabela 1 mostra algumas características das cidades estudadas.

Tabela 1. Características gerais das cidades selecionadas para estudo da variabilidade pluviométrica.

\begin{tabular}{|c|c|c|c|c|}
\hline Município & Altitude & Latitude & Longitude & Período \\
\hline Orós & $188 \mathrm{~m}$ & $6^{\circ} 15^{\prime} 00^{\prime \prime}$ & $38^{\circ} 55^{\prime} 00^{\prime \prime}$ & $2000-2010$ \\
\hline Várzea Alegre & $311 \mathrm{~m}$ & $6^{\circ} 48^{\prime} 00^{\prime \prime}$ & $39^{\circ} 18^{\prime} 00^{\prime \prime}$ & $2000-2010$ \\
\hline Iguatu & $217 \mathrm{~m}$ & $6^{\circ} 22^{\prime} 00^{\prime \prime}$ & $39^{\circ} 18^{\prime} 00^{\prime \prime}$ & $2000-2010$ \\
\hline Quixelô & $208 \mathrm{~m}$ & $6^{\circ} 14^{\prime} 00^{\prime \prime}$ & $39^{\circ} 11^{\prime} 00^{\prime \prime}$ & $2000-2010$ \\
\hline Icó & $148 \mathrm{~m}$ & $6^{\circ} 24^{\prime} 00^{\prime \prime}$ & $38^{\circ} 51^{\prime} 00^{\prime \prime}$ & $2000-2010$ \\
\hline Cariús & $315 \mathrm{~m}$ & $6^{\circ} 32^{\prime} 00^{\prime \prime}$ & $39^{\circ} 30^{\prime} 00^{\prime \prime}$ & $2000-2010$ \\
\hline Jucás & $246 \mathrm{~m}$ & $6^{\circ} 31^{\prime} 00^{\prime \prime}$ & $39^{\circ} 31^{\prime} 00^{\prime \prime}$ & $2000-2010$ \\
\hline Tarrafas & $281 \mathrm{~m}$ & $6^{\circ} 41^{\prime} 00^{\prime \prime}$ & $39^{\circ} 45^{\prime} 00^{\prime \prime}$ & $2000-2010$ \\
\hline Lavras da M. & $279 \mathrm{~m}$ & $6^{\circ} 45^{\prime} 00^{\prime \prime}$ & $38^{\circ} 58^{\prime} 00^{\prime \prime}$ & $2000-2010$ \\
\hline
\end{tabular}




\begin{tabular}{ccccc} 
Baixio & $493 \mathrm{~m}$ & $6^{\circ} 44^{\prime} 00^{\prime \prime}$ & $38^{\circ} 43^{\prime} 00^{\prime \prime}$ & $2000-2010$ \\
\hline Umari & $47 \mathrm{~m}$ & $6^{\circ} 38^{\prime} 00^{\prime \prime}$ & $38^{\circ} 42^{\prime} 00^{\prime \prime}$ & $2000-2010$ \\
\hline Ipaumirim & $262 \mathrm{~m}$ & $6^{\circ} 47^{\prime} 00^{\prime \prime}$ & $38^{\circ} 43^{\prime} 00^{\prime \prime}$ & $2000-2010$ \\
\hline Ant.do Norte & $365 \mathrm{~m}$ & $6^{\circ} 47^{\prime} 00^{\prime \prime}$ & $39^{\circ} 59^{\prime} 00^{\prime \prime}$ & $2000-2010$ \\
\hline Cedro & $250 \mathrm{~m}$ & $6^{\circ} 36^{\prime} 00^{\prime \prime}$ & $39^{\circ} 04^{\prime} 00^{\prime \prime}$ & $2000-2010$ \\
\hline
\end{tabular}

O período do estudo foi de 2000 a 2010. Para a geração da média da década da precipitação de cada cidade foi feita uma média aritmética das precipitações anuais do período de estudo no software Microsoft Excel 2010.

Posteriormente, foi gerada uma planilha com valores de $\mathrm{X}, \mathrm{Y}$ e $\mathrm{Z}$ (latitude, longitude, e total precipitado na década, respectivamente) para interpolação dos dados e modelagem superficial no programa estatístico Surfer e, em seguida, a espacialização no software ArcVIEW e Arc/INFO versão 9.3 através do método de interpolação por krigagem e semivariograma linear. Foi gerado ainda um gráfico que representa as médias das décadas e a média geral de todas as cidades, na perspectiva de mostrar quais cidades estão acima/abaixo da média estimada.

\section{RESULTADOS E DISCUSSÃO}

Na Figura 2, está representada a espacialização da precipitação média decadal de cada cidade no período de estudo $(2000$ - 2010). Nela, podemos observar que as cidades localizadas na região central do Centro Sul do Ceará tendem a ter um maior valor médio da precipitação, com o seu pico máximo na cidade de Iguatu, com valor médio de 1019,4 $\mathrm{mm}$. Resultado semelhante para esta média foi encontrado por Silva et al. (2014) estudando a variabilidade das chuvas no município de Iguatu e os impactos dos eventos El Niño e La Niña.

Nessa figura, ainda, pode-se constatar a dissimilaridade das precipitações na região, fator bem característico do semiárido brasileiro, onde as precipitações são mal distribuídas no espaço e tendem a ser irregulares durante todo tempo (GUERREIRO, 2013). A cidade que obteve valor mínimo para as precipitações foi a cidade de Cariús $(650,4 \mathrm{~mm})$. A espacialização da precipitação é de grande valia para a elaboração de projetos de irrigação em áreas específicas e no armazenamento de água precipitada, que segundo Araújo (2003) e Malveira (2009) correspondem a 93\% de toda água ofertada aos usuários.

Tem importância também a ideia de que quando se tem um grande espelho d'água não necessariamente a evaporação da água contribuirá para a precipitação na mesma região, e o exemplo claro disso é o município de Orós, que é detentor de um dos maiores açudes do estado com capacidade 2.100.000.000 $\mathrm{m}^{3}$, mas que tem uma precipitação muito inferior à média das outras cidades $(679 \mathrm{~mm})$. 


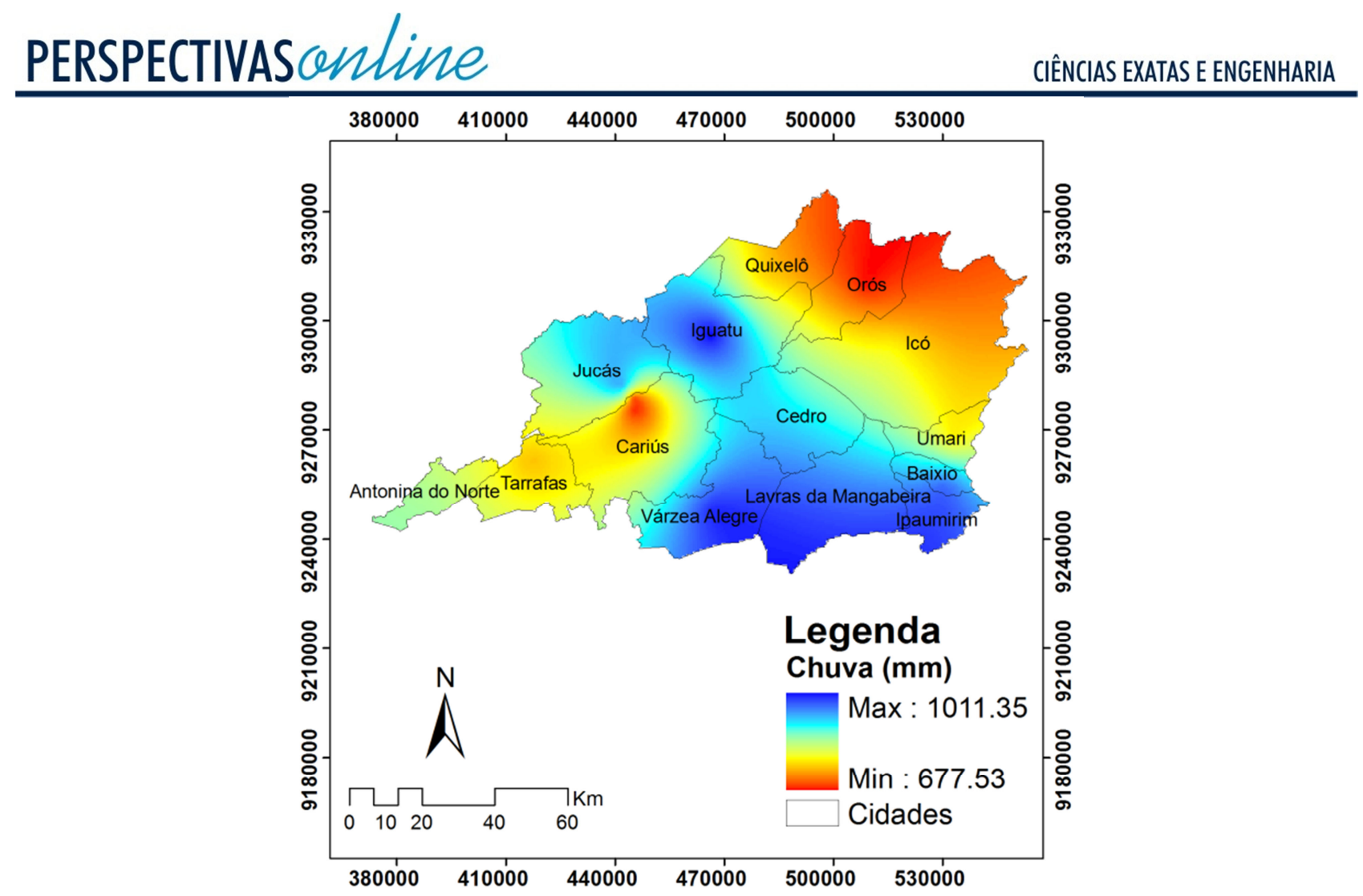

Figura 2. Precipitações médias anuais das cidades do Centro Sul do Ceará no período de 2000 - 2010.

Fonte: Elaboração própria.

Analisando a Figura 3, observa-se a distribuição da precipitação pluviométrica média das décadas dos municípios analisados. Verifica-se pelo regime da distribuição que há inúmeras oscilações dentro de uma região relativamente pequena em termos nacionais, e que grande parte das cidades sofrem com a estiagem e baixos índices pluviométricos. Não somente o estado do Ceará, mas também toda a região Nordeste do Brasil sofre com a crise hídrica, e com isso o Estado criou alternativas de convício com esse tipo de clima, sendo um dos líderes em captação e armazenamento de água.

Outro fator que influência nas precipitações sazonais na região Nordeste do Brasil é a Zona de Convergência Intertropical (ZCIT) e os eventos meteorológicos La Niña e El Niño (MOLION E BERNARDO, 2002). Segundo Pereira (2011), estudando a variação da precipitação no município de Caicó$\mathrm{RN}$, observou que em todos os acontecimentos do evento El Niño, cerca de $50 \%$ dos eventos apresentaram precipitação abaixo da média anual. Porém esses eventos que influenciam na precipitação não ocorrem sozinho, sendo necessário um conjunto de fatores que favoreçam essa influência na precipitação. 


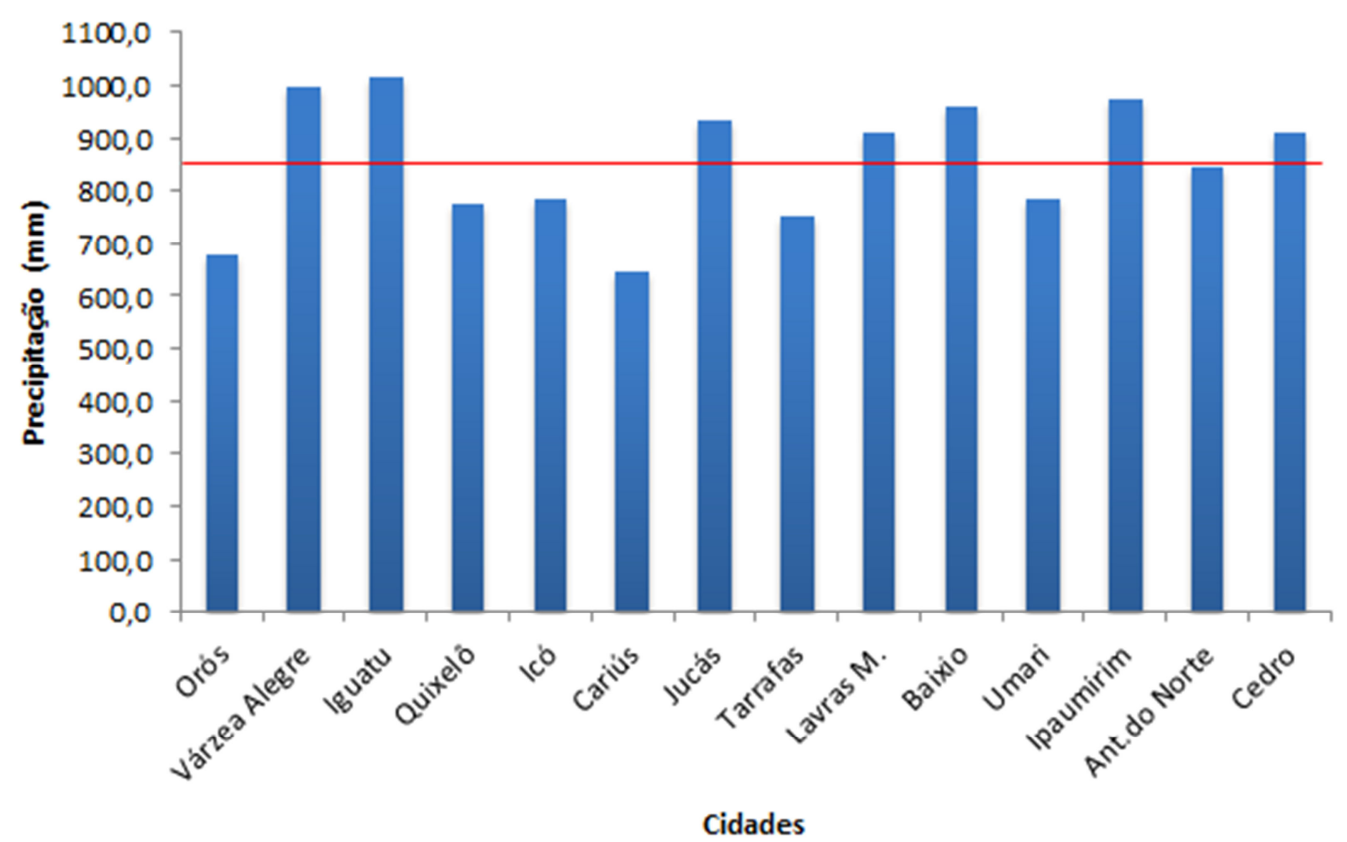

Figura 3. Distribuição das precipitações médias anuais do Centro Sul do Ceará no período de 2000 - 2010. Fonte: Elaboração própria.

Ainda com relação à Figura 3, municípios como Orós, Icó, Quixelô, Cariús, vêm sofrendo com a grande estiagem refletida nos últimos anos, o que tem impacto nos diversos setores da economia. Já cidades como Iguatu, Várzea Alegre, Jucás, têm bons valores médios de precipitação. Contudo, não devem descuidar no que diz respeito ao armazenamento de água, tendo em vista que a seca é um fator característico do clima semiárido.

\section{CONCLUSÕES}

O regime de precipitação das cidades analisadas apresentou oscilações durante todo o estudo. As cidades de Iguatu e Várzea Alegre apresentaram os maiores valores médios de precipitação, 1019,4 e 1001,7 $\mathrm{mm}$, respectivamente. Já as cidades de Cariús e Orós apresentaram os menores valores médios de precipitação, com 657 e $688 \mathrm{~mm}$, respectivamente, evidenciando a grande estiagem sofrida na região nesta última década. Os softwares utilizados auxiliaram para melhor evidenciar os municípios que estão sofrendo com a estiagem. É necessário um maior número de políticas públicas para a região, tendo em vista um baixo índice pluviométrico na última década e a "seca" como fator predominante da região.

\section{REFERÊNCIAS}

ARAI, F. K.; GONÇALVES G. G. G.; PEREIRA S. B.; COMUNELlO E.; VITORINO A. C. T.; DANIEL O. Engenharia Agrícola, Jaboticabal, v.30, n.5, p.922-931, set./out. 2010.

ARAÚJO, J. C. D. Assoreamento em Reservatórios do Semi-árido: Modelagem e Validação. Revista Brasileira de Recursos Hídricos, v. 8, n. 2, p. 13, 2003.

BEZERRA, A. C. N., ROCHA, E. J. P., ROLIM, P. A. M. Identificação da região do El- Niño que influencia com maior intensidade o regime de precipitação no litoral leste da Amazônia através das anomalias de TSM do Oceano Pacífico. In: XIII Congresso Brasileiro de Agrometeorologia, 2003, santa Maria-RS. Situação atual e perspectivas da Agrometeorologia, 2003. v. 2, p. 1037-1038. 
FIETZ, C.R.; URCHEI, M.A.; COMUNELLO, E. Probabilidade de ocorrência de chuva na bacia do rio Dourados - MS. Dourados: Embrapa Agropecuária Oeste, 2002. 32 p.

FREITAS, A. J.; SILVA, D. D.; PRUSKI, F. F.; PINTO, F. A.; PEREIRA, S. B.; GOMES FILHO, R. R.; TEIXEIRA, A. F.; BAENA, L. G. N.; MELLO, L. T. A.; NOVAES, L. F. Equações de chuvas intensas no Estado de Minas Gerais. Belo Horizonte: Companhia de Saneamento de Minas Gerais; Viçosa: Universidade Federal de Viçosa, 2001. 65 p.

GUERREIRO M. J. S; ANDRADE, E. M.; ABREU, I; LAJINHA, T. Long-term variation of precipitation indices in Ceará State, Northeast Brazil. International Journal of Climatology, v. 33, n. 14, p. 2929-2939, November, 2013

HASTENRATH, S., 1984: Interannual variability and annual cycle: mechanisms of circulation and climate in the tropical Atlantic. Mon. Wea. Rev., v.112, p.1097-1107

MAGALHÃES, A. R.; GLANTZ, M. H., 1992: Socioeconomic impacts of climate variations and policy responces in Brazil, United Nations Environment Program (UNEP), Secretariat for Planning and Coordination State of Ceara (SEPLAN), Esquel Brasil Foundation, $155 \mathrm{pp}$.

MALVEIRA, V. T. C. Pequena açudagem e sustentabilidade hidrológica em grandes bacias semiáridas: estudo de caso da bacia do açude Orós. Dissertação de mestrado, (Engenharia civil, área de concentração recursos hídricos) Departamento de Engenharia Hidráulica e Ambiental. 2009.

MARINS, A. P. P.; E. M. C. D.; TASSI, R. A influência do assoreamento na variação dos níveis do Reservatório do Vacacaí-Mirim / Santa Maria - RS. VI Encontro Nacional de Engenharia de Sedimentos, v. 1, p. 1-4, 2004.

MOLION, L. C. B.; BERNARDO, S. O. Uma revisão da dinâmica das chuvas no nordeste brasileiro. Revista Brasileira de Meteorologia, v.17, n.1,1-10, 2002.

PEREIRA, V. C.; SOBRINHO, J. E.; OLIVEIRA, A. D.; MELO, T. K.; VIEIRA, R. Y. M. Influência dos eventos el niño e la niña na precipitação pluviométrica de Mossoró-RN. Enciclopédia Biosfera, v.7, n. 12, 2011.

RIBEIRO DA SILVA, K.; PAIVA, Y.G.; CECÍLIO, R.A.; PEZZOPANE, J. E. M. Avaliação de interpoladores para a espacialização de variáveis climáticas na bacia do rio Itapemirim - ES. In: SIMPÓSIO BRASILEIRO DE SENSORIAMENTO REMOTO, 2007, Florianópolis. Resumos... São José dos Campos: INPE, 2007. p. 3. 141-3. 146. Press, 2005.

ROGERS, P. P.; LLAMAS, M. R.; CORTINA, L. M. (Ed.). Water crisis: myth or reality? CRC

SILVA, F. J. A.; ARAÚJO, A. L.; SOUZA, R. O. Águas subterrâneas no Ceará - poços instalados e salinidade. Revista Tecnologia, Fortaleza, v.28, n.2, p.136-159, 2007.

SILVA, E. B.; ARAÚJO NETO, J. R.; BRASIL, J. B.; GUEDES, T. A.; BANDEIRA, D. M. F.. Estudo da precipitação pluviométrica no município de Iguatu-CE. Congresso de Pesquisa e Inovação da rede Norte e Nordeste de Educação Tecnológica - IX CONNEPI, 2014.

SOMLYODY, L.; VA RIS, O. Freshwater under pressure. International Review for Environmental Strategies, v.6, n.2, p.181-204, 2006. 